\title{
The value of nasal mupirocin in containing an outbreak of methicillin-resistant Staphylococcus aureus in an orthopaedic unit
}

\author{
S. P. Barrett \\ Microbiology Department, Southend Hospital, Westcliff-on-Sea, Essex \\ SSO ORY, UK. \\ Accepted for publication 3rd October 1989
}

\begin{abstract}
Summary: An outbreak of methicillin-resistant Staphylococcus aureus (MRSA) occurred in two adjacent orthopaedic wards following the admission of a known carrier. The outbreak was not contained by ward closure or by standard infection control measures. Eventually several nasal carriers were identified and treated with nasal mupirocin, following which the outbreak ended.
\end{abstract}

Keywords: MRSA; mupirocin; hospital outbreak.

\section{Introduction}

In many areas of south-east England, methicillin-resistant Staphylococcus aureus (MRSA) is now the most noticeable example of cross-infection within hospital and the most prevalent form is EMRSA-1 referred to as the Thames epidemic strain (Marples, Richardson \& de Saxe, 1986). This organism is endemic in the hospitals of Southend Health District (Barrett $e t$ al., 1988), and in so far as infection control precautions are possible, these are based on guidelines prepared by a working party of the North-East Thames Regional Microbiology Subcommittee (Adhami et al., 1987). These guidelines recommend that individuals affected with MRSA should be treated with mupirocin, a potent antistaphylococcal agent for topical use (Casewell \& Hill, 1987). Mupirocin has been found effective in the elimination of MRSA when other topical agents such as chlorhexidine have been unsuccessful (Hill, Duckworth \& Casewell, 1988). This report of MRSA in an orthopaedic unit discusses the measures used to contain an outbreak and demonstrates the role of nasal mupirocin. 


\section{Patients and Methods}

\section{Investigation of the outbreak}

Two adjacent orthopaedic wards admitting patients of either sex over the age of $14 \mathrm{yrs}$ were involved. The outbreak followed the admission of a patient known to be colonized with MRSA (Figure 1, patient 1). Although this patient was source-isolated in a side-room, a second case (patient 2) was discovered 19 days later. In accordance with the Regional guidelines, all staff and patients were then screened for MRSA. Cotton-tipped swabs were used for screening of potential carriage sites and were moistened with sterile distilled water before application to both anterior nares, both axillae or the perineum. Where applicable, swabs were also taken from skin lesions, and samples of catheter urine and sputum were examined. In the case of subjects found positive for MRSA, a blood agar plate was touched against the scalp hair to check for carriage in this site. Six swabs from carriage sites or from urine/sputum were streaked on to a blood agar plate and also on to mannitol-salt agar (Oxoid), the Oxford staphylococcus was streaked on to each plate as a control. A filter-paper disk containing $25 \mu \mathrm{g}$ methicillin (Mast Laboratories) was overlaid on the mannitol-salt agar and both plates were incubated at $37^{\circ} \mathrm{C}$ for 36 hours. Colonies of staphylococci appearing resistant to methicillin were identified using the 'Staphaurex' slide agglutination test. Suspect colonies of MRSA were then tested in pure culture against a methicillin strip using the Oxford staphylococcus as a control. Strains growing less than $20 \mathrm{~mm}$ from the strip were considered methicillin-resistant. Other antibiotic susceptibilities were determined by the Stokes method (Stokes \& Waterworth, 1972).

The affected ward was closed when more than two patients were found to be carrying MRSA, and thereafter only MRSA-positive patients from adjacent ward areas were admitted to it. Screening of all staff and patients was repeated four weeks later.

\section{Control measures}

Each patient found to be MRSA-positive was treated in accordance with the Regional guidelines. Patients were isolated either singly or in four-bedded side wards. Small areas of broken skin were treated with mupirocin cream; larger wounds were treated with aqueous chlorhexidine. Chlorhexidine was used to wash the hair if affected, and skin carriage was treated by daily bathing with triclosan bath concentrate; patients who could not use a bathroom were given bed baths with aqueous chlorhexidine. Mupirocin nasal ointment ( $2 \%$ in paraffin base), provided for use in clinical trials by Beecham Pharmaceuticals, Brentford, Middlesex, was applied to both anterior nares twice daily for five days in cases of nasal carriage. Antibacterials were stopped after one week of suppressive treatment and the patient was checked for recrudescence of MRSA by reswabbing 3, 6 and 9 days later. When a ward area had been cleared of MRSA patients it was thoroughly cleaned before re-use. The cleaning protocol included washing 
with a $2 \%$ phenolic disinfectant of all beds, mattress and pillow covers, ward furniture, level surfaces, floors and walls; all linen and curtains were replaced. Bathrooms and toilets were treated with a chlorine-releasing cleaning powder.

\section{Results}

Figure 1 shows the results of swab examination for MRSA for all patients who were positive at any time during the outbreak. The initial screening of the ward, undertaken after the second patient was detected, revealed two additional MRSA-positive patients (patients $3 \& 4$ ). No staff member was found positive. Despite the application of infection control measures, a review of the situation in the middle of August, approximately four weeks following ward closure, gave no indication that the outbreak was being controlled since of eight patients who had been treated repeatedly with suppressive measures, seven had yielded positive specimens within the previous week. Only one patient, who had received nasal mupirocin for nasal carriage (patient 4), had been cleared of MRSA, and had remained clear for approximately five weeks. Following a repeat screening another seven cases (patients 9-15) were then detected, five of these had positive nasal swabs and in four this was the sole site of colonization. These patients were given suppressive treatment including topical or nasal mupirocin where indicated. Nasal mupirocin was also given to patient 6 who now showed a positive nasal culture for the first time, and to patient 8 whose

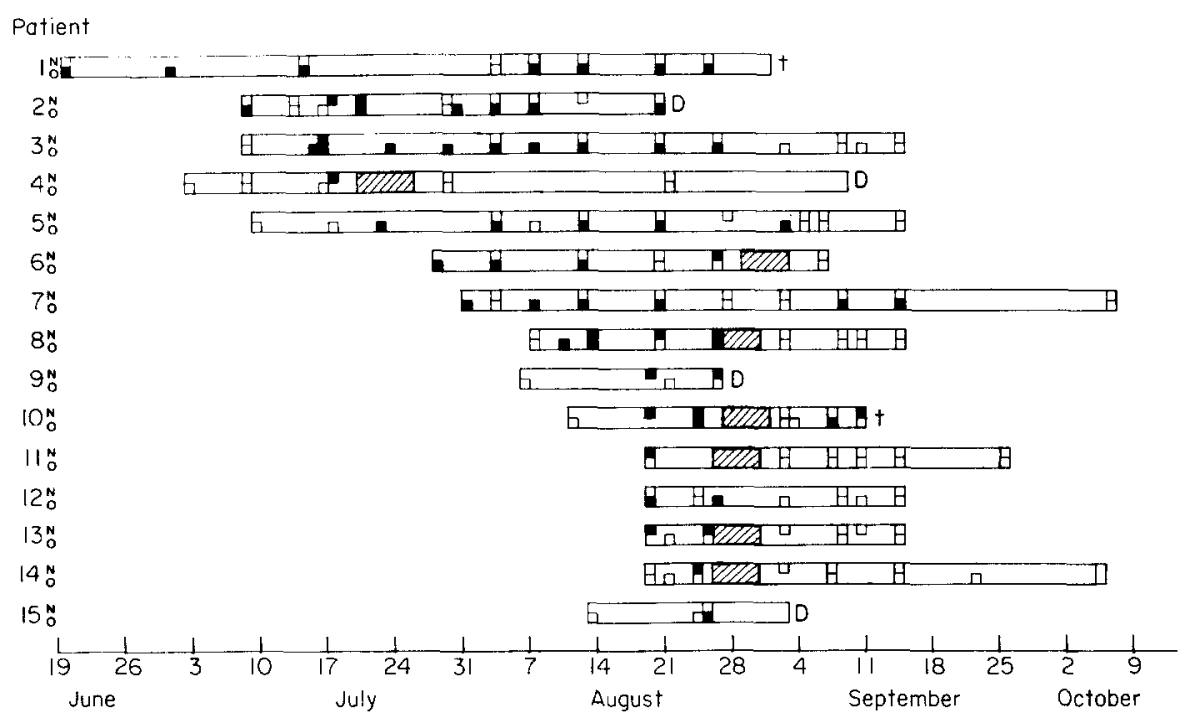

Figure 1. Swab results from EMRSA patients. $N=$ nose swab; $O=$ swab from extranasal sites; $\square$ positive for EMRSA; $\square$ negative for EMRSA; $\mathbb{Z}=$ treatment with nasal mupirocin; $\mathrm{D}=$ discharged $\boldsymbol{\dagger}=$ died. 
Table I. Isolation of MRSA from extranasal sites of patients with and without nasal MRSA*

\begin{tabular}{lcc}
\hline Site of extranasal MRSA & $\begin{array}{c}\text { Number of patients } \\
\text { with nasal MRSA }\end{array}$ & $\begin{array}{c}\text { Number of patients } \\
\text { without nasal MRSA }\end{array}$
\end{tabular}

\begin{tabular}{lll}
\hline Operative wound & 5 & 3 \\
Pressure sore & 1 & 3 \\
Axilla & 2 & 0 \\
Perineum & 3 & 1 \\
Scalp hair & 1 & 1 \\
Total patients & 5 & 5 \\
\hline
\end{tabular}

*Patients yielding MRSA from nasal swabs only are not included.

nasal colonization had inadvertently been left untreated for two weeks; patient 9 was discharged before swab results were available. Patients $1,5,7$, 12 and 15 never yielded MRSA from nasal culture, the distribution of extranasal sites from which MRSA was isolated is shown in Table I.

Following the widespread introduction of nasal mupirocin on 26 August, only three patients yielded positive specimens; patient 10 who was terminally ill, and patients 5 and 7 who had MRSA in extranasal sites and both of whom later became MRSA-negative. Swabs were taken from affected patients at a similar rate both before $(0.63$ per patient-day) and after $(0.72$ per patient-day) this date and the overall proportion of those yielding MRSA decreased from 60/216 to 7/179. No new case of MRSA was detected after the introduction of nasal mupirocin and the ward reopened three weeks later after a total of eight weeks closure.

\section{Discussion}

This orthopaedic unit had a long mean patient-stay of 17.7 days, and it was necessary for MRSA-positive patients to remain on the ward for long periods after detection. The difficulties posed by a large number of patients requiring infection control measures may have been responsible for the accidental omission of nasal mupirocin from some regimes, although all other suppressive measures were applied correctly. The first four patients to be detected were isolated and received appropriate suppressive treatment apart from patients 2 and 3 whose nasal carriage was inadvertently left untreated. Patients 58 who were discovered later by routine swabs were treated similarly along with the earlier patients, although again one (patient 8) did not receive nasal mupirocin promptly. Patients 9-15 had been MRSA-positive for an unknown period prior to the second staff and patient screening on 19 August. The end of the outbreak coincided with the death or discharge of patients 1,2 and 9 and the detection and treatment of patients $9-15$ as well as with the introduction of nasal mupirocin and it is therefore impossible to be certain of the role played by the use of nasal 
mupirocin. However, since standard infection control measures had proved ineffective in the early stages of the outbreak, it seems likely that the failure to control the outbreak was related to inadequate treatment of nasal MRSA in identified patients, and the presence of unidentified nasal carriers; the problem may have been compounded by transient nasal carriage which was shown by at least two patients ( 2 and 3 ). This outbreak was controlled although enrichment culture was not used when examining for MRSA colonization; enrichment culture has been found to contribute little to the control of at least one outbreak (Hill et al., 1988).

The propensity for patient-to-patient spread shown by the Thames epidemic MRSA has resulted in many hospital outbreaks (Marples \& Cooke, 1985) and guidelines for its control have been produced by working parties of the Hospital Infection Society/British Society for Antimicrobial Chemotherapy (Ayliffe et al., 1986) and of the North East Thames Regional Health Authority (Adhami et al., 1987). The value of nasal mupirocin in the elimination of Staphylococcus aureus was demonstrated by Casewell and Hill (1986) and this agent has been included in both the above sets of guidelines. Nasal mupirocin was considered of value in the control of ward and hospital outbreaks of MRSA by Dacre, Emmerson \& Jenner (1986) and by Hill et al. (1988), and in the control of an outbreak of MRSA in an endemic situation in a Special Care Baby Unit (Davies et al., 1988).

In these and other reports the agent was used, as in the present study, as one of a number of infection control measures likely to have aided control of spread of MRSA, although Hill et al. (1988) also noted a reduction in extranasal MRSA following the use of nasal mupirocin. The particular interest of the present report is that the number of patients involved continued to increase despite standard treatment until several nasal carriers were discovered and then treated with nasal mupirocin. After this time the outbreak rapidly came under control and it therefore seems likely that this agent played an important part, over and above that of standard measures, in terminating the outbreak.

I am grateful for the advice and assistance of Professor A. M. Emmerson and Mrs J. Catton.

\section{References}

Adhami, Z., Barrett, S., Bradley, J., Duckworth, G., Gill, N., Milne, S., 'Teare, E. L. \& Thompson, R. (1987). Report of the North East Thames Microbiology Sub Committee MRSA Working Party. North East Thames Regional Health Authority.

Ayliffe, G. A. J., Duckworth, G. J., Brumfitt, W., Casewell M. W.. Cooke, E. M., Cookson, B. D., Keane, C. T., Marples, R. R., Phillips, I., Shanson, D. C., Simmons, N. A. \& Williams, J. D. (1986). Guidelines for the control of epidemic methicillin-resistant Staphylococcus aureus. Fournal of Hospital Infection 7, 193-201.

Barrett, S. P., Gill, O. N., Mellor, J. A., \& Bryant, J. C. (1988). Methicillin-resistant Staphylococcus aureus in linked district hospitals. Postgraduate Medical Fournal 64, 606-609. 
Casewell, M. W. \& Hill, R. L. R. (1986). Elimination of nasal carriage of Staphylococcus aureus with mupirocin ("pseudomonic acid") - a controlled trial. Fournal of Antimicrobial Chemotherapy 17, 365-372.

Casewell, M. W. \& Hill, R. L. R. (1987). Mupirocin ("pseudomonic acid") - "a promising new topical antimicrobial agent". Fournal of Antimicrobial Chemotherapy 19, 1--5.

Dacre, J., Emmerson, A. M. \& Jenner E. A. (1986). Gentamicin-methicillin-resistant Staphylococcus aureus: epidemiology and containment of an outbreak. Yournal of Hospital Infection 7, 130-136.

Davies, E. A., Emmerson, A. M., Hogg, G. M., Patterson, M. F. \& Shields, M. D. (1987). An outbreak of infection with a methicillin-resistant Staphylococcus aureus in a special care baby unit: value of topical mupirocin and of traditional methods of infection control. Fournal of Hospital Infection 10, 120-128.

Hill, R. L. R., Duckworth, G. J. \& Casewell, M. W. (1988). Elimination of nasal carriage of methicillin-resistant Staphylococcus aureus with mupirocin during a hospital outbreak. Yournal of Antimicrobial Chemotherapy 22, 377-384.

Marples, R. R. \& Cooke, E. M. Workshop on methicillin-resistant Staphylococcus aureus held at the headquarters of the Public Health Laboratory Service on 8 January 1985. Yournal of Hospital Infection 1985, 6, 342-348.

Marples, R. R., Richardson, J. F. \& de Saxe, M. J. (1986). Bacteriological characters of strains of Staphylococcus aureus submitted to a reference laboratory related to methicillin resistance. Journal of Hygiene (Cambridge) 96, 217-223.

Stokes, E. J. \& Waterworth P. M. (1972). Association of Clinical Pathologists Broadsheet No. 55 (revised). 